\title{
Use of Sharesource in Remote Patient Management in Peritoneal Dialysis: A UK Nurse's Perspective
}

\author{
Michelle Blaauw
}

Great Ormond Street Hospital for Children NHS Foundation Trust, London, UK

\begin{abstract}
In June 2015, Sharesource, a cloud-based, bi-directional connectivity platform, was launched by Baxter Healthcare in the UK. With this came the ability to use remote patient management (RPM) with peritoneal dialysis (PD) patients in an easy, standardized manner across dialysis units. Improved nursing efficiency and patient outcomes, achieved utilizing RPM include individualized PD patient prescriptions, early detection and prevention of potential PD complications, reduced planned and unplanned hospital attendance, reduced drop off, and increased healthcare professional and patient/carer confidence in self-care. These are illustrated with Case Studies. The potential to reduce glucose exposure is explored and for PD units yet to use RPM, lessons learned during the implementation of Sharesource are shared.
\end{abstract}

(c) 2019 S. Karger AG, Base

\section{Background}

In the UK, there are 63,162 adult patients receiving renal replacement therapy (RRT), with $40 \%$ on hemodialysis and only $6 \%$ on peritoneal dialysis (PD; Renal Registry 2018) [1]. In pediatrics, $11 \%$ of the RRT patients receive PD and $12 \%$ receive hemodialysis, from a total of 964 patients [2]. The total UK PD population ( 3,895 patients) in 2018 remains very small compared to those on hemodialysis (25,379 patients), and although patients receiving RRT each year is grow- 
ing in the UK, this increase is mostly seen in those with a functioning transplant (5.1\%) and those receiving hemodialysis (0.9\%), with PD seeing less than a $0.1 \%$ growth [1]. Finding new ways to increase the number of patients receiving home $\mathrm{PD}$, alongside reducing the number of patients "dropping off" PD is urgently required, to address this large disparity in adult RRT management. There are lessons to be learnt from pediatric renal units in the UK, where there are good dialysis decision pathway discussions in low clearance clinics, a home first approach to therapies and acute start PD and assisted PD are available. Remote patient management (RPM) systems, like Sharesource, have the potential to address these issues and support tailored patient care.

In the UK, most home PD programs are nurse led. The essential roles of the PD nurse include:

- Pre-dialysis education and home assessment

- Education of the patient and/or carers to carry out dialysis independently

- Psychological support of patient and family to manage home PD, outpatient clinic reviews - planned and unplanned

- PD prescription management

- Coordination of PD patient care between community and hospital

- Assessment of dialysis adequacy and assessment of long-term PD complications [3].

Optimizing patients' quality of life is a central tenant of PD nursing, with a focus on preventing patient drop off and reducing hospitalizations for patients. Prior to RPM, supporting PD patients at home consisted of frequent phone calls to those most vulnerable, PD nurse home visits, and regular hospital appointments for patients. PD prescription changes would routinely take place at the time of the clinic visit, onto the patient's pro card (a memory card that could store and upload programs onto their device at home and from which PD treatment data could be downloaded), or a home visit would be arranged to make this PD change in the patient's home.

When Sharesource, a cloud-based, bi-directional connectivity platform was launched in the UK, it provided PD nurses with detailed dialysis data on a daily basis, of all PD patients. The ability to view each patient's weight, blood pressure, ultrafiltration (UF) data, along with a detailed PD summary, including full cycle visibility and an alarm log, brought with it an opportunity to review and reassess PD nursing practice. The platform also has the ability to enable PD prescription changes remotely, by the PD nurse at the hospital directly through to the individual patient's PD device at home. 


\section{How to Implement RPM/Sharesource onto a PD Unit?}

Four important questions need to be addressed prior to implementing RPM: "Who will be responsible for monitoring the RPM?," "Which patients should have RPM?," "Which safety settings/flag rules should be set?," and "How frequently will Sharesource be monitored?"

\section{Who Will Be Responsible for Monitoring the RPM?}

Each unit needs to decide if this should be the PD nurses' responsibility or the nephrologist's responsibility. In the UK, Sharesource monitoring and management falls mostly on PD nurses as part of their daily role, with the nephrologists utilizing the platform during outpatient clinics and multidisciplinary team meetings.

Be careful not to share this responsibility between too large a team, as there is a risk of work duplication. For PD units with large patient numbers, named patients are best allocated under a specific nurse or doctor, to prevent the potential for work duplication and to share the monitoring load. Local decisions also need to be made, as to who on the unit gets access to Sharesource and who can implement PD changes.

\section{Which Patients Should Have RPM?}

If the goal of RPM is to improve patient outcomes, it only feels right that all home PD patients be offered the choice of Sharesource. However, a phased roll out may be advised if the PD unit is large. If too large a patient cohort is started at once, there is the potential to be overwhelmed by too much data initially. For a phased approach, the decision needs to be made as to which patients should be given RPM first, that is, which patients will most benefit from the visibility of Sharesource? - newly trained patients? those with complex comorbidities? patients who are frequently hospitalized or exhibit high risk behaviors?

\section{Which Safety Settings ("Flag Rules" and "Priority Values") Should Be Set?}

Safety settings or "flag rules" need to be set within Sharesource to notify the PD team each day of any PD problems experienced by patients at home. These flag rules are set for all patients within a PD unit, they are not patient-specific. The SHARESOURCE platform is available on the latest Baxter PD machines. With the AMIA device, available in the USA and Canada, there are 13 flag rules available, while there are 8 flag rule settings on the HOMECHOICE CLARIA - the PD device available across Europe.

Once flag rules have been chosen, a priority value needs to be set for each as these will trigger a yellow (priority) or red (high priority) flag, if reached. The 
Table 1. Flag rules guide

\begin{tabular}{|c|c|c|c|}
\hline Flag rules & $\begin{array}{l}\text { Treatment } \\
\text { adherence } \\
\text { concerns }\end{array}$ & $\begin{array}{l}\text { Poor drainage/ } \\
\text { possible catheter } \\
\text { malfunction }\end{array}$ & $\begin{array}{l}\text { Volume } \\
\text { manage- } \\
\text { ment issues }\end{array}$ \\
\hline Adjusted peritoneal volume & $x$ & & \\
\hline Events/alerts during treatment & & $x$ & \\
\hline Blood pressure not within range $(A)$ & & & $x$ \\
\hline Deviations during treatment $(\mathrm{A})$ & $x$ & & \\
\hline Drain ended early & $x$ & $\mathrm{X}$ & $x$ \\
\hline \multicolumn{4}{|c|}{ Excessive drain volume/high drain occurred } \\
\hline Lost dwell time & & $\mathrm{X}$ & \\
\hline Lost therapy volume & $x$ & & \\
\hline Lost therapy time & $x$ & & \\
\hline Night UF not as estimated (A) & & $x$ & $x$ \\
\hline Weight not within target range $(\mathrm{A})$ & & $\mathrm{X}$ & $x$ \\
\hline Initial drain variance & $x$ & $x$ & $x$ \\
\hline
\end{tabular}

A, AMIA device only flag rule.

flags displayed each day on the Sharesource Dashboard quickly help the clinicians to detect potential issues in each patient, in 3 key areas: patient concordance issues, poor drainage /possible catheter malfunction, and volume management concerns (Table 1).

\section{How Frequently Will Sharesource Be Monitored?}

When RPM is first rolled out to patients, some initial time investment into Sharesource is advised, to understand how best to use and optimize its full potential for each patient.

Checking the RPM platform at the start of each shift is now a common practice for PD nurses in the UK. This daily check of the dashboard enables the team to assess all patients quickly and prioritize the day ahead, through rapid recognition of patients in need of a review.

\section{Patient Education}

Once an implementation strategy has been agreed, RPM can be put into practice. This needs to include planned patient and carer education of the PD device, which incorporates Sharesource connectivity. Patients should be informed during this education session, how frequently the PD team will be reviewing Sharesource. It is essential that PD patients and carers understand that they still have the responsibility to call the unit with any PD problems or if they are feeling unwell. Our unit found some initial complacency when Sharesource was launched, as patients and carers perceived that the PD nurses could now "see everything." 


\section{RPM: Impact on Nursing Practice and How It Can Be Used to Improve Patient Outcomes}

When Sharesource was launched in January 2016 at Great Ormond Street Hospital, the PD nurses' use of time quickly changed. The historical practice of phoning the most "vulnerable" patients on a sometimes daily basis was no longer required, as now each patient's weight, blood pressure, and UF data were available on Sharesource. The time invested into monitoring Sharesource was found to be more effective compared to phoning patients for this data; often removing the need for costly interpretation services, previously required for some patients. $\mathrm{Pa}$ tients were still telephoned at times, but the nature of these phone calls changed; they were now being made to give positive reinforcement of self-care activity, to give feedback on the data seen on Sharesource, or to modify the patient's PD program or laxative management, for example, in response to a bad night of alarms. Both patients and carers at home reported feeling more supported, knowing that the nurses had visibility of their PD at home and clinicians felt more confident to discharge some of the more complex or vulnerable patients home, knowing their daily treatment regime, weight and blood pressure could be monitored remotely.

Having made the decision to give all patients RPM, the nursing team were in an exceptional position to monitor all patients consistently, not just those deemed most vulnerable. Daily monitoring of the Sharesource Dashboard enabled quick detection of missed treatments, and any event flags helped warn the team of potential problems that occurred overnight that may need nursing attention. This warning system and the detailed daily treatment summaries, available through RPM, led to the better utilization of nursing time. The ability to modify each patient's PD prescription remotely, in response to the data seen daily on Sharesource, quickly changed practice. As a result, a $73 \%$ increase in remote $\mathrm{PD}$ prescription changes were recorded per patient per month $(0.11-$ 0.19 , Fig. 1) in a 6-month period [4]. PD prescription changes were now being made in a proactive manner, addressing early problems seen, instead of waiting for patients to call or turn up unplanned on the unit with drain problems, with patient's prescriptions becoming more tailored as a result [5]. These findings are in accordance with Firanek et al. [6], Pharro and Lewis [7] and Patterson [8] who have all demonstrated that UK PD nurse activities have become more proactive and less reactive, since the introduction of Sharesource. Pharro and Lewis [7] report that this ability to be more proactive in their treatment of patients led to a reduction in patients leaving PD from $63 \%$ of patients in 2015 pre RPM to only $29 \%$ in 2016 with RPM. The need for less home visits was reported, with the ability to support patients now remotely with Sharesource, leading to an increase in total PD patient numbers, with no additional nurse resource required. 


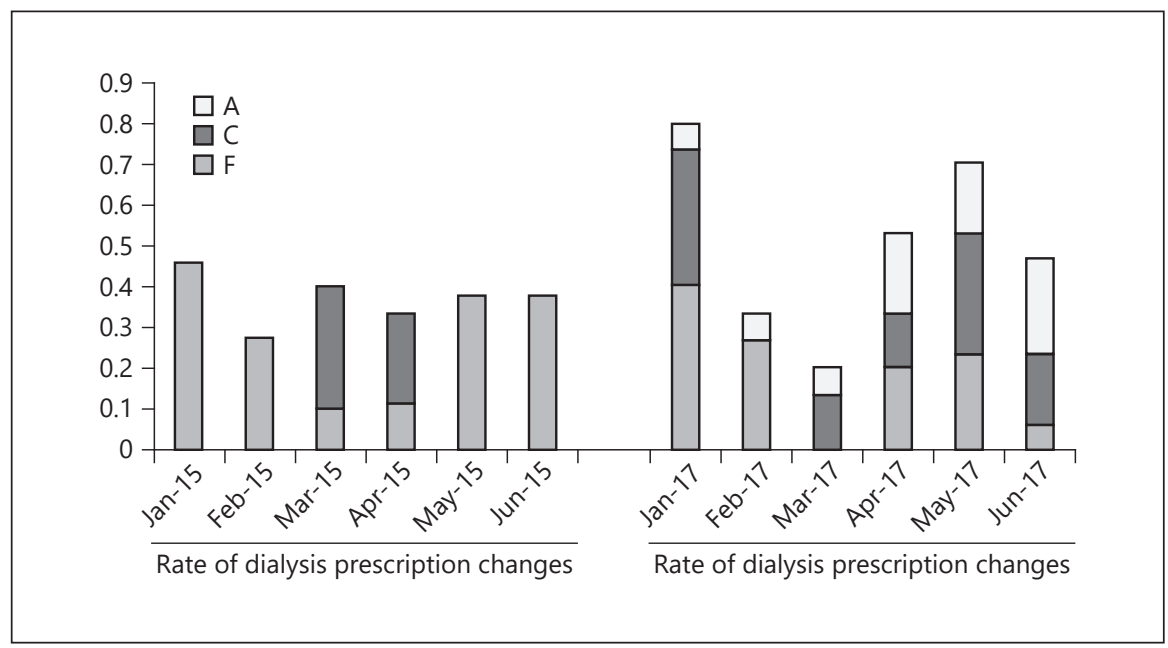

Fig. 1. Rate of dialysis prescription changes made remotely pre- and post-implementation (mean number of prescription changes per patient per month), including changes to alarm settings (A), number of cycles (C), and fill volume (F).

The daily monitoring of data on Sharesource gives the nurse a better insight into how well the patient's PD catheter is working, how well the patient is coping/managing on home PD, how burdened the patient is with overnight alarms, how compliant the patient may be with their prescribed PD program and may help when interpreting poor dialysis adequacy results. This detailed monitoring can be especially important for new patients on $\mathrm{PD}$, as it enables the nurse to identify and address these potential issues quickly, before they become too great a burden for the new patient. This daily RPM monitoring of new patients in their first 3 months on therapy, when the potential for trouble shooting issues are most prevalent, can led to PD prescriptions being more "tailor-made" and personalized for patients [9].

\section{Individualized PD Prescriptions/Optimizing Patient Drains}

At Great Ormond Street Hospital, there has been a move away from using the device default drain settings for all patients, in response to the drain data seen on Sharesource. The result is more individualized prescriptions for patients, as illustrated by case study A [4].

Case Study Example: Patient A, an adolescent male who was already obese before starting PD. His PD fill volume was $2.0 \mathrm{~L} 1.36 \%$ bags, with Extraneal last bag fill. The standard PD device settings of $85 \%$ minimum drain and no last fill manual drain UF target were used. Patient A had a good working PD catheter and was not constipated. 


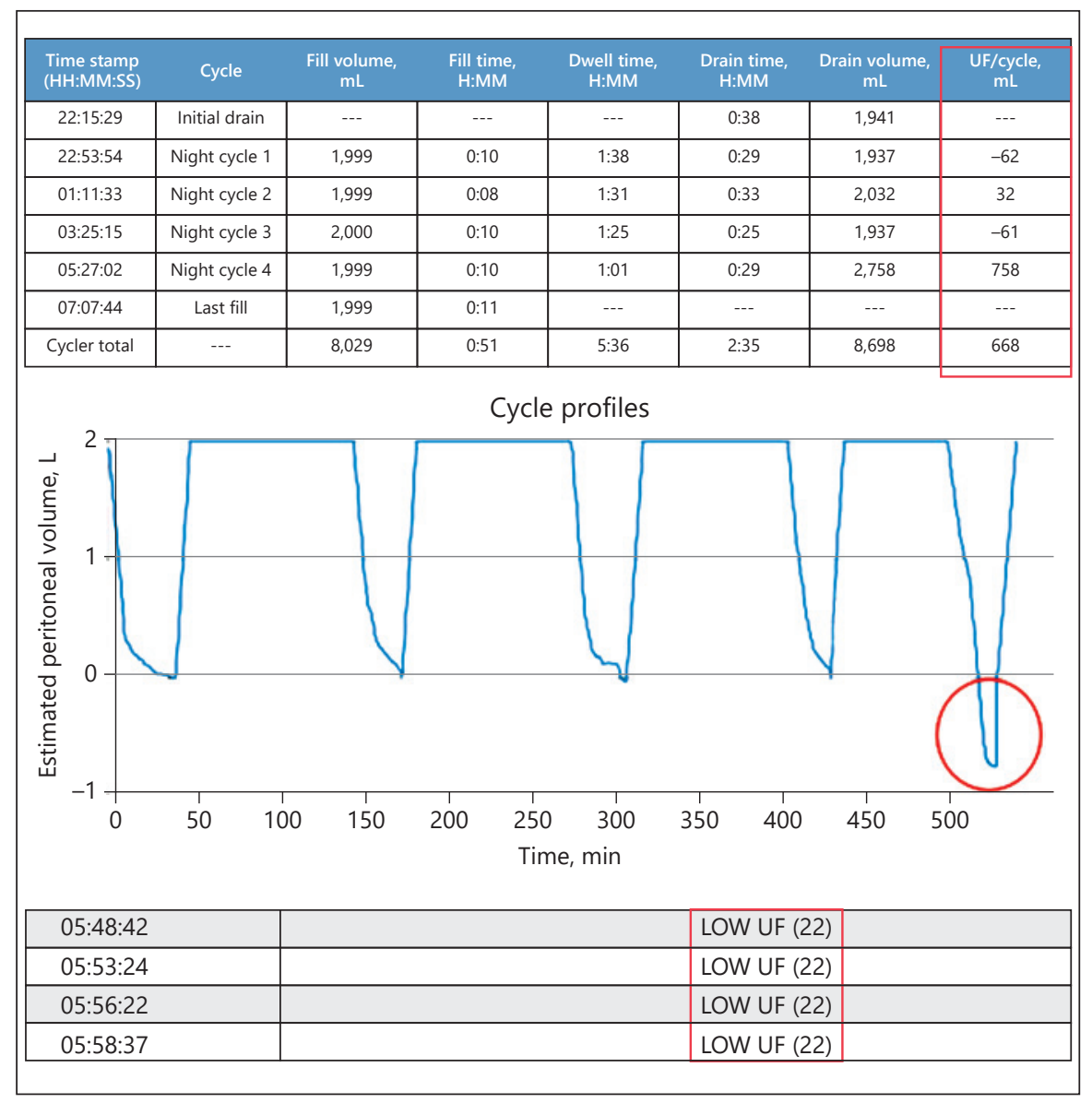

Fig. 2. Sharesource daily treatment summary. UF, ultrafiltration.

After Patient A's first week on established PD, his UF trend ranged from 27 mLs up to $614 \mathrm{mLs}$ over a 6-day period. Patient A reported no drain alarms overnight.

On reviewing Sharesource, it was revealed that patient A often retained PD fluid on 2 or 3 cycles each night, but that the volume of this pocketed fluid was not sufficient to trigger the default $85 \%$ drain alarm.

Some nights, this fluid would be pocketed early into the dialysis session, allowing time for it to drain out overnight, through change of his sleep position. While on other nights, if this fluid was pocketed towards the end of the PD session, the therapy would finish with this fluid still inside patient $A$, in addition to the patient A's Extraneal last bag fill. On the night when the patient's net UF was only $27 \mathrm{mLs}$, Sharesource showed that patient A retained $278 \mathrm{mLs}$ on his last 
drain (drain alarm would have been triggered if $300 \mathrm{mLs}$ was retained), prior to his last fill.

Prior to RPM, this patient would have needed intermittent use of $2.27 \%$ bags to correct regular fluid absorption and resulting fluid overload. However, being able to understand why his UF trend was so variable, led the team to implement 2 prescription changes: to increase his minimum drain alarm to $95 \%$ and to set a last fill manual drain UF target of $400 \mathrm{mLs}$.

The following week, still using all $1.36 \%$ bags, patient A's UF trend became more consistent, ranging from 412 to $669 \mathrm{mLs}$. Six months later - patient A had continued the same program. He avoided the need for intermittent $2.27 \%$ bags, continued to have stable consistent UF trends and prevented additional weight gain related to $\mathrm{PD}$.

Figure 2 demonstrates how effective a last fill manual drain UF target can be - in this example, the patient is an anuric adolescent, who was on track to have a net negative UF for the night, had the UF target of $600 \mathrm{mLs}$ not been set. Both of these examples demonstrate how Sharesource is used to improve patient drains overnight. The potential impact is a reduction in strong glucose bag usage for patients.

\section{Constipation Management}

An excellent example of how RPM has enabled more proactive nursing practice is in its use to guide laxative management. Being notified of a "lost dwell time" and/or "events/alerts during treatment" flag on the Sharesource Dashboard and then being able to review the patient's drain data, over a few days to see if there is a worsening drain pattern, can help the nurse identify if the problem is bowel related. The usual uniform, consistent cycle profile changes to an irregular, inconsistent one (Fig. 3). This data enables the PD nurse to increase a patient's laxatives, often before the patient has identified that they are constipated. Being proactive in this way, has been demonstrated to reduce the number of unplanned hospital visits $[8,10]$. At Great Ormond Street Hospital, since the introduction of RPM in Jan 2016, there has been no PD catheter changes secondary to constipation-related catheter migration [4].

\section{Clinic Appointments and Hospitalizations}

In some units, the use of RPM with PD patients and the subsequent shift to proactive nursing care, by utilization of remote changes to patients' regimes, has resulted in a reduction in planned hospital clinic appointments [4, 11]. At Great Ormond Street Hospital, this was taken a step further with the introduction of a nurse-led telemedicine clinic. The telemedicine clinic was developed and utilized with the more stable PD patients, on an alternate clinic basis. The telemed- 


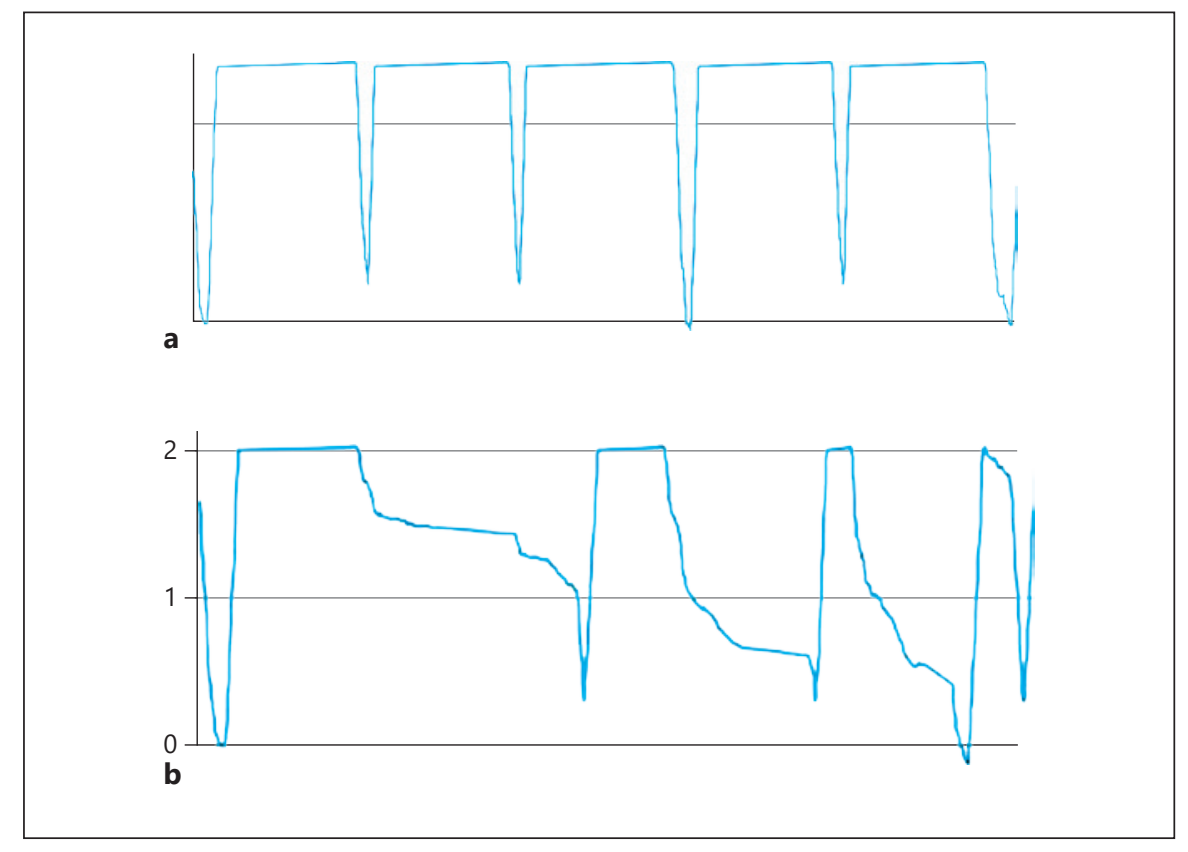

Fig. 3. Cycle profiles. a Tidal PD with a good working catheter. b Impact of constipation.

icine review was routinely a detailed telephone review, although for some patients a video link (Facetime) was possible. The patient or carer emailed their PD record chart to the $\mathrm{PD}$ nurse on the morning of the planned telemedicine clinic visit, for prior review. If there were any concerns about the appearance of the PD exit site, a photo of this is also sent. The structure of the telemedicine clinic followed the same structure as a clinic appointment (except physical examination), but offered longer and more flexible appointment times, with more time available before the consultation to focus on the patient's Sharesource data and prescription management. Although this virtual clinic was not appropriate for all patients (those with complex comorbidities or those patients with language barriers were still seen regularly in person), a resultant $20 \% /$ patient/month reduction (1.2-1.0) in clinic attendance occurred over a 6 month period, with no adverse effect on patient safety [4]. These clinics were well received by patients and carers, with less missed school/work and reduced transport costs for families being welcomed.

Studies in both the UK and Columbia have shown a reduction in PD-related hospitalizations, since RPM has been introduced $[10,12,13]$. However, further study is required to look at the potential impact RPM could have on peritonitis rates, but small patient examples have already been reported showing acute re- 
ductions in UF seen on Sharesource prior to peritonitis episodes, which could potentially lead to earlier detection and treatment of peritonitis in the future [14, $15]$.

\section{The Future of RPM}

As Sharesource continues to evolve and improve, the potential for improved PD patient outcomes grows with it. In early 2019, Baxter ADEQUEST, the windows-based tool used to measure, report, and model PD prescriptions for individual patients, commonly used by PD nurses, will be incorporated into the platform, enabling nurses to input adequacy, standard PET and min PET data and model prescription outcomes for individual patients. This will only help to streamline the nursing practice further.

\section{Summary}

Although there has been very little research, carried out to date, on the impact of RPM on PD patient outcomes, this chapter has helped to demonstrate how UK nursing practice has evolved with the use of Sharesource. The move to more proactive nursing care, seen through case study examples, has led to more personalized PD prescriptions, early detection of potential PD problems, and reduced hospital attendance. The potential for Sharesource to reduce PD glucose exposure, patient drop off, hospitalization and peritonitis rates, whilst increasing patient numbers without additional nursing resource, is worthy of exploration.

\section{References}

1 MacNeill S, Ford D, Evans K, Medcalf J: UK Renal Registry 20th Annual Report: Chapter 2 UK renal replacement therapy adult prevalence in 2016: National and centre-specific analyses. Nephron 2018;139(suppl 1):47-74.

2 Plumb L, Wong E, Casula A, Braddon F, Lewis M, Marks S, Shenoy S, Sinha M, Maxwell H: UK Renal Registry 20th Annual Report: Chapter 4 demography of the UK paediatric renal replacement therapy population in 2016. Nephron 2018; 139(suppl 1):105-116.

3 Levy J, Morgan J, Brown E: Oxford Handbook of Dialysis (ed 3). Oxford, Oxford University Press, 2009.
4 Blaauw M, Barber A: Management of children requiring peritoneal dialysis at great Ormond street hospital: introduction of a remote patient management platform. Arch Dis Child 2018; 103(suppl 2):A22.

5 Blaauw M: Continuous quality improvement: a cloud based platform for remote peritoneal dialy sis paediatric patient management. Perit Dial Int March 2017;37(suppl 1):S14-S21.

6 Firanek C, Knowles M, Sloand J: The impact of Automated Peritoneal Dialysis with Remote Patient Management: Changing the Nursing Paradigm to Proactive Clinical Management, EDTA 2017, https://www.abstracts2view.com/era_archive/view.php?nu=ERA17L1_1496. 
7 Pharro G, Lewis S: Optimizing Nursing Resource and Time Efficiency with Remote Monitoring in Automated Peritoneal Dialysis Patients. Dublin, Euro PD, 2017.

8 Patterson T: Remote Patient Monitoring in Peritoneal Dialysis: Evaluating the Benefits of Implementation on Service Resource. Dublin, Euro PD 2017.

9 Milan Manani S, Crepaldi C, Giuliani A, Virzi GM, Garzotto F, Riello C, De Cal M, Rosner MH, Ronco C: Remote monitoring of automated peritoneal dialysis improves personalization of dialytic prescription and patient's indpendence. Blood Purif 2018;46:111-117.

10 Chukwu C, MacGlashan A, Chandrasekar T, Abraham A: Peritoneal Dialysis in the Cloud: the use of a Cloud Based Remote Monitoring System in Peritoneal Dialysis Therapy, EDTA 2017, https://www.abstracts2view.com/era_archive/ view.php?nu=ERA17L1_2546.

11 McCarthy K, Roper-Knowles M: Using a Remote Two-Way Patient Management System to Tailor APD Therapy, UKKW 2018 P366. https://www. ukkw.org.uk/abstracts-from-uk-kidney-week/.
12 Rivera A, Sanabria M, Vesga J, Suarez A, Bunch A: Comparison of Hospitalization Rate in Automated Peritoneal Dialysis Patients with and without Remote Management Program in Colombia, ASN 2018. https://www.asn-online.org/education/kidneyweek/2018/program-abstract. aspx? controlId=3018350.

13 Milan Manani S RM, Virzì GM, Giuliani A, Berti S, Crepaldi C and Ronco C: Longitudinal experience with remote monitoring for automated peritoneal dialysis patients. Nephron 2019, in Press.

14 Rojas-Diaz M, Ramos A: Help of Remote Patient Monitoring in the Assessment of Changes in Ultrafiltration Before, During and after a Peritonitis Episode in Patients on Automated Peritoneal Dialysis, ASN 2017. https://www.asn-online.org/ education/kidneyweek/2017/program-abstract. aspx? controlId=2784136.

15 Gomez R, Petro C, Dazzarola M, Rivera A: Early Suspicion of Peritonitis with APD Cycle-Embedded Remote Patient Management Technology with Sharesource. Dublin, Euro PD, 2017.

Michelle Blaauw

Great Ormond Street Hospital for Children NHS Foundation Trust

Great Ormond Street

London, WC1N 3JH (UK)

E-Mail michelle.blaauw@gosh.nhs.uk 